https://doi.org/10.7203/Normas.3.4675

\title{
SECUENCIAS PONDERADORAS DE IDENTIFICACIÓN CON COMO EN TEXTOS LITERARIOS Y PERIODÍSTICOS CUBANOS (2000-2008)
}

\author{
EMPHATIC SEQUENCES OF IDENTIFICATION WITH COMO IN LITERARY \\ TEXTS AND NEWSPAPER IN CUBAN (2000-2008)
}

PEDRO DE JESÚS LÓPEZ ACOSTA Casa de Cultura de Fomento (Cuba)

\section{RESUMEN:}

La presente investigación analiza, a partir de textos cubanos actuales, un tipo de construcciones que proponemos denominar «secuencias ponderadoras de identificación con como». Se explica su funcionamiento en calidad de construcción lingüística peculiar mediante la descripción e interpretación de sus características morfosintácticas y semánticas.

PALABRAS CLAVE: partícula como, ponderación, énfasis, identificación, ejemplificación, referencia, preposición, secuencias ponderadoras, secuencias de identificación, gramática, lingüística, semántica.

\section{ABSTRACT:}

By examining current Cuban texts, this study analyzes a type of constructions which are proposed to be denominated "emphatic sequences of identification with como". It tries to explain these sequences as a peculiar linguistic construction by means of the description and interpretation of their morphosyntactic and semantic characteristics.

KEY WORDS: particle como, emphasis, identification, exemplification, reference, preposition, emphatic sequences, sequences of identification, grammar, linguistics, semantics. 


\section{INTRODUCCIÓN}

La presencia en español de las construcciones que aquí se denominan «secuencias ponderadoras de identificación con como» es de larga data, a juzgar por los ejemplos literarios rastreados en la bibliografía teórica de consulta, procedentes del Quijote: «¿Piensa vuestra merced caminar este camino en balde, y dejar pasar y perder un tan rico y tan principal casamiento como este, donde le dan en dote un reino?» (apud Cuervo, 1998: 229); o de otros muchos textos de los siglos XIX, XX y XXI citados por la Real Academia de la Lengua Española (RAE) en su Nueva gramática de la lengua española (NGLE): «El hombre, que a todos los animales domina, que de todos se vale, que se alimenta con los más nobles ¿temblará ante un indigno roedor como tú?» (apud NGLE, 2010: 374); «Con un obispo enfermo, y enfermo como este, iba pudriéndose la diócesis» (apud NGLE, 2010: 566); «En un país como el nuestro nos aferramos a las palabras, con todo lo que tienen de mísero y luminoso» (apud NGLE, 2010: 712).

Sin embargo, los estudios lingüísticos en lengua española apenas han reparado en este tipo de secuencias, y cuando han emprendido su análisis lo han hecho situándolas entre las construcciones comparativas, modal-comparativas o relativas con como.

La recurrente interpretación de estas secuencias como parte -muchas veces marginal- de estructuras sintácticas más generales de la lengua ha impedido describir y explicar, de manera objetiva, las características morfosintácticas y semánticas que las hacen un fenómeno peculiar dentro de los variados usos de como.

Hasta las postrimerías del pasado siglo, en el año 2000, no aparece un trabajo dedicado específicamente a secuencias de como emparentadas con las ponderadoras de identificación: ¿Qué hace un chicarrón como tú en un sitio como éste?, de Salvador Gutiérrez Ordóñez. Las construcciones analizadas allí poseen un notorio carácter ponderador, pero resulta cuestionable su función identificadora; y no solo porque el autor las interprete como estructuras atributivas adscriptivas o denotadoras de clase, sino porque los ejemplos con que trabaja son creaciones artificiales y carecen de un contexto oracional y textual adecuado para definir con exactitud el alcance de la referencia que establecen.

A Gutiérrez Ordóñez le corresponde, no obstante, el incuestionable mérito de emprender un estudio independiente de realizaciones próximas a estas secuencias, e incluso de haber fundamentado teóricamente su distinción respecto de las construcciones comparativas con como. En su análisis insiste en uno de los tópicos más socorridos de la tradición lingüística hispánica en relación con muchas de las realizaciones de esta partícula: la presunción de estructuras verbales elípticas -en este caso, del copulativo ser-, y la consiguiente adjudicación del valor relativo a como.

NORMAS. REVISTA DE ESTUDIOS LINGÜÍSTICOS HISPÁNICOS, NÚMERO 3 (AÑO 2013):

http://www.uv.es/normas

(ISSN 2174-7245) 
Todos los autores ${ }^{1}$, por tangenciales que sean sus análisis de las construcciones aquí llamadas «secuencias ponderadoras de identificación con como», han debido enfrentarse a repetidos problemas:

a) El verbo copulativo implícito o elíptico que puede, en ciertos enunciados, explicitarse o expandirse.

b) La naturaleza de la partícula como, cuya categoría gramatical (adverbio relativo, preposición o conjunción) se supedita a la interpretación del segmento que introduce como elíptico o no elíptico, y al tipo de construcción sintáctica más general dentro de la cual se analiza (oraciones comparativas, comparativas modales, relativas).

c) La especificidad o inespecificidad de la referencia que establece la secuencia con como en el enunciado donde se inserta y los distintos valores que, consecuentemente, adquiere (comparación, adscripción en una clase, identificación, ejemplificación, etc.).

d) La inclusión o exclusión de las construcciones con presencia del correlato tan...como en el análisis de aquellas que no lo poseen.

Dada la carencia de estudios cubanos al respecto -que se suma a la escasez y parcialidad de los emprendidos en el ámbito hispanohablante en general, así como a la insuficiente base de análisis con realizaciones lingüísticas concretas-, consideramos de interés realizar esta investigación, orientada a la descripción y explicación de estas secuencias a partir de textos literarios y periodísticos cubanos actuales.

Se muestrearon cuarenta y un documentos: doce textos literarios (escritos por autores cubanos y publicados en la Isla entre los años 2000 y 2007) y veintinueve números de publicaciones periódicas (aparecidas entre los meses de marzo y junio del año 2008). Los textos literarios comprenden los géneros de novela, cuento y ensayo (cuatro libros de cada uno) y los periodísticos veinticuatro números de diarios nacionales (Granma y Juventud Rebelde) y cinco del periódico provincial de Sancti Spíritus (Escambray).

De un total de 2464 páginas útiles se extrajeron 4509 realizaciones de como, de las cuales solo 116 constituían secuencias ponderadoras de identificación con como.

\footnotetext{
${ }^{1}$ Véanse, fundamentalmente, además del artículo de Gutiérrez Ordóñez (2000), los textos de Cano Aguilar (1995), Alcina y Blecua (1994), Martí Sánchez (1996 y 1999), Iglesias Bango (2006) y Jiménez Juliá (2003).
}

NORMAS. REVISTA DE ESTUDIOS LINGÜÍSTICOS HISPÁNICOS, NÚMERO 3 (AÑO 2013):

http://www.uv.es/normas

(ISSN 2174-7245) 


\section{DEFINICIÓN Y CRITERIOS DE EXCLUSIÓN}

Se denomina secuencias ponderadoras de identificación con como a las construcciones objeto de estudio porque:

a) Constituyen una clase particular de construcciones de orden invariable, integradas por dos sintagmas nominales: el primero -de mayor significado extensionaldenota la clase a que pertenece el segundo -ligado entonativamente con aquel-, que posee mayor significado intensional ${ }^{2}$ y aparece introducido por la partícula como.

b) La extensión referencial del primer sintagma se hace mínima para identificarse o igualarse con la extensión referencial del sintagma introducido por como, de manera que se establece entre ambos una correferencia estricta.

c) La secuencia, de carácter enfático, encarece o pondera una cualidad -positiva o negativa, implícita o explícita-, de la clase denotada por el primer sintagma expresándola como inherente al sintagma introducido por como.

Estas características se manifiestan a través de dos restricciones morfosintácticas fundamentales: la concordancia obligada entre ambos sintagmas de la secuencia y la imposibilidad de que esta aparezca actualizada con el valor identificador del artículo.

Pero la más importante propiedad de estas secuencias, desde el punto de vista teórico-metodológico, es la relativa al carácter de la correferencia, y alcanza a serlo gracias a su valor discriminatorio. Ella nos permitió excluir del estudio las secuencias que no establecían una identidad referencial estricta entre sus sintagmas básicos, aun cuando tuviesen carácter ponderador, sus constituyentes se atuvieran a la estructura invariable descrita y observaran las restricciones morfosintácticas fundamentales.

Sucedió así con secuencias en las cuales como encabeza enumeraciones de elementos unidos asindética o disyuntivamente, hecho que connota la no exhaustividad de los miembros enumerados y refuerza la correferencia no estricta entre estos y el sintagma que precede a como:

1) Sus ideas y entusiasmo se han regado empíricamente por todo el lomerío y hoy alcanzan a SITIOS COMO LA CUQUITA, CAIMANES, LA BIJA, LIMONCILlO ARRIBA... y llegan hasta Guisa, en la hermana provincia de Granma (Juventud 5).

${ }^{2}$ Se sigue el criterio de Lyons (1973: 467): «La extensión de un término consiste en la clase de entidades a las que el término se aplica o refiere; la intensión de un término es el conjunto de atributos que caracteriza a cualquier entidad a la cual se aplica correctamente el término. La extensión y la intensión varían inversamente en sus relaciones mutuas: cuanto más grande es la extensión de un término, menor es su intensión, y viceversa».

NORMAS. REVISTA DE ESTUDIOS LINGÜÍSTICOS HISPÁNICOS, NÚMERO 3 (AÑO 2013):

http://www.uv.es/normas

(ISSN 2174-7245) 
2) La Cumbre Estados Unidos-Rusia se cerró con la firma de un acuerdo-marco estratégico que fija las líneas maestras de la relación bilateral para el futuro en ÁREAS COMO LA LUCHA CONTRA EL TERRORISMO O LA ECONOMÍA (Granma, abril 7: 4).

En este uso de la conjunción disyuntiva con interpretación inclusiva (RAE § 31.4.1a) se manifiesta «la llamada elección abierta, en la que las opciones mencionadas se presentan como ejemplo de las posibles» (2010: 614). Otro tanto sucede con el empleo de expresiones textuales cuyo cometido lingüístico es específicamente ese:

3) [...] no se puede producir o dar servicios a todo costo, eso no lo resiste la economía de nuestro país que ofrece miles de SERVICIOS GRATUITOS COSTOSOS COMO LA EDUCACIÓN Y LA SALUD, POR CITAR SOLO DOS (Granma, mayo 23: 10).

4) En sus páginas aparecen FIRMAS TAN PRESTIGIOSAS COMO LAS DE NICOLÁS GUILLÉN, JuAN MARINELlo, JesÚs ORTA RUIZ (El INDIO NABORÍ), MANUEL NAVARRo LuNA Y ANA NÚÑEZ MACHÍN, ENTRE OTROS MUCHOS INTELECTUALES DE NUESTRO PAÍS (Granma, mayo 16: 3).

Por otra parte, cuando la partícula introduce una enumeración en que aparece el nexo copulativo y como elemento de cierre, pueden darse dos situaciones: las unidades enumeradas se presentan, inobjetablemente, como los únicos miembros de su clase que afectan la predicación del enunciado; o se produce cierta ambigüedad, similar a la de otros contextos de la lengua donde la conjunción y es intercambiable por la conjunción $o$ (y viceversa) en enumeraciones abiertas (RAE $\S 31.4 .1 \mathrm{~b})$ :

5) [...] el evento cuenta con un jurado presidido por el maestro Hugo Oslé, cantante y director artístico, e integrado por PRESTIGIOSAS FIGURAS COMO HÉCTOR TÉLLEZ, Migdalia Hechavarría, Elizabeth D'Gracia, RaQuel HernándeZ, ZENaida ARMENTEROS, ZOA FERNÁNDEZ, SUSANA JUNCO, RIDULFO VAILlANT Y JORGE NÚÑEZ (presidentes de la UNEAC en Santiago de Cuba y Guantánamo) (Granma, junio, 6: 12).

6) [...] los habitantes de este sureño territorio [...] saludan la epopeya de Girón con acciones muy concretas en LUGARES TAN RECÓNDITOS COMO COCODRILO, GUASASA, SANTO TOMÁS Y VÍNCULO, caseríos casi en el anonimato en épocas pasadas (Granma, abril, 9: 2).

En virtud de la extensión de la enumeración en el ejemplo 5, así como de la naturaleza de la referencia que establece el enunciado (relación oficial de los miembros del jurado de un concurso), su exhaustividad es más notoria que en 6 , donde pudiera interpretarse la ausencia de otros miembros de la clase lugares muy recónditos. Sin

NORMAS. REVISTA DE ESTUDIOS LINGÜÍSTICOS HISPÁNICOS, NÚMERO 3 (AÑO 2013):

http://www.uv.es/normas

(ISSN 2174-7245) 
desconocer hechos como estos, y hasta tanto se perfilen métodos de discriminación más certeros, en esta investigación se considerará ambos tipos de secuencias como ponderadoras de identificación, teniendo en cuenta que la selección del nexo copulativo $y$ por oposición al disyuntivo $o$ puede constituir una marca atendible del interés que tiene el emisor de presentar la serie en correferencia estricta con el primer sintagma de la secuencia ${ }^{3}$.

Mucho más engorrosos resultaron los deslindes en ciertos casos donde el sintagma encabezado por como presenta un solo sustantivo (o unidad equivalente), en singular o plural. Se trata de secuencias y enunciados referencialmente ambiguos en tanto no hay una voluntad marcada del emisor por distinguir si la predicación afecta específica y exclusivamente a la unidad presentada con como o si la afecta de manera prototípica. Solo en la primera variante la referencia del sintagma que precede a como puede hacerse mínima y coincidir estrictamente con la referencia del otro sintagma básico de la secuencia. En la segunda variante ya esto no es viable, bien porque la referencia del sintagma que antecede a como puede ampliarse en un grado máximo para incluir a todos los miembros de la clase que denota (alcance universal), bien porque tiene la posibilidad de indefinirse para incluir, indistintamente, a cualesquiera de los miembros de la clase (referencia aleatoria) ${ }^{4}$ :

7) Te pregunta si no oyes al perro por la noche [...] Luego te comenta que a él no lo deja dormir, desde hace unos meses no para de ladrar por gusto [...] UN PERRO COMO ESE VALE, pero por cien dólares se lo entrega a cualquiera (Minuciosas 20).

8) ¿Por qué no pensaba en Amalia Touza?, dijo de sopetón, «la anciana beata a quien despojaste de sus joyas en la calle Obispo». Únicamente UNA VIEJA CATÓLICA, APOSTÓLICA Y ROMANA COMO ELLA podía escribir CARTAS COMO LAS QUE YO RECIBÍA.

${ }^{3}$ Cfr. notas 9 y 10 .

${ }^{4}$ En el ejemplo 7 son igualmente plausibles las interpretaciones específica y exclusiva -que ese perro específico (y solo él) vale-; y también la prototípica de alcance universal -que todos los perros de ese tipo valen-. Por su parte, en el ejemplo 8, que reúne dos secuencias, se dan varias opciones que combinan las interpretaciones específica y exclusiva y la prototípica de referencia aleatoria:

a) Únicamente ella (y solo ella), Amalia Touza -que es una vieja católica, apostólica y romana-es capaz de escribir las cartas específicas (y solo esas) que el personaje recibe.

b) Únicamente ella (y solo ella), Amalia Touza -que es una vieja católica, apostólica y romana- es capaz de escribir cartas (cualesquiera) del tipo de las que el personaje recibe.

c) Únicamente una vieja (cualquiera) del tipo de Amalia Touza -católicas, apostólicas y romanases capaz de escribir las cartas específicas (y solo esas) que el personaje recibe.

d) Únicamente una vieja (cualquiera) del tipo de Amalia Touza -católicas, apostólicas y romanases capaz de escribir cartas (cualesquiera) del tipo de las que el personaje recibe.

NORMAS. REVISTA DE ESTUDIOS LINGÜÍSTICOS HISPÁNICOS, NÚMERO 3 (AÑO 2013):

http://www.uv.es/normas

(ISSN 2174-7245) 
Nadie que no conociera los textos bíblicos los citaría con tanta exactitud ni tendría la ocurrencia de escribir cartas semejantes (Cándido 148).

Obviamente, este tipo de secuencias se excluyó del corpus. Asimismo, las realizaciones ponderadoras de identificación con como fueron sometidas a otra prueba: la supresión del primer sintagma básico junto con la partícula ${ }^{5}$. Esta elisión no altera la referencia del enunciado, hecho que reafirma el cometido fundamental de la construcción: identificar el sintagma encabezado por como:

9) En UN PAís COMO LOS ESTAdOS UNIDOS, los conceptos de «melting pot»y «multiculturalism» han querido dar cuenta, con limitaciones, del fenómeno de su diversidad poblacional y cultural (Diálogos 126).

En LOS ESTADOS UNIDOS, los conceptos de «melting pot» $\mathrm{y}$ «multiculturalism» han querido dar cuenta, con limitaciones, del fenómeno de su diversidad poblacional y cultural.

La posibilidad, casi general, de suprimir el primero de los sintagmas básicos más como se halla en estrecha consonancia con la imposibilidad, también casi general, de suprimir la(s) unidad(es) introducida(s) por la partícula:

*En un país los conceptos de «melting pot»y «multiculturalism» han querido dar cuenta, con limitaciones, del fenómeno de su diversidad poblacional y cultural.

En solo pocos ejemplos de la muestra pueden suprimirse estas unidades sin menoscabar ostensiblemente la referencia: cuando han sido implícita o explícitamente presentadas en el contexto previo y el primer sintagma adquiere la capacidad de referirlas anafóricamente en virtud de la sinonimia contextual que establecen:

${ }^{5}$ Las únicas secuencias privadas de esa posibilidad son aquellas en que como introduce un elemento anafórico (pronombre demostrativo en función sustantiva u otra(s) unidad(es) sustantivada(s) por la anteposición del artículo): «El sentido de huida a la vida, y, sobre todo, la imagen de la fuga como fluir vital del mundo, aparecen con especial fuerza en VERSOS COMO ESTOS DE "NOCHE...BLANCURA": "Palomas. Fugas. Nadas, amanecidas, trémulas [...]" (Saturno 123). [*El sentido de huida a la vida, y, sobre todo, la imagen de la fuga como fluir vital del mundo, aparecen con especial fuerza en estos de «Noche...blancura»] «[...] los latinoamericanistas debaten el uso de un marco discursivo que no siempre se adapta (si se adopta) en UN CONTEXTO COMO EL LATINOAMERICANO (Diálogos 25). [*Los latinoamericanistas debaten el uso de un marco discursivo que no siempre se adapta (si se adopta) en el latinoamericano]

NORMAS. REVISTA DE ESTUdIOS LINGÜÍSTICOS HISPÁNICOS, NÚMERO 3 (AÑO 2013):

http://www.uv.es/normas

(ISSN 2174-7245) 
10) Durante este período, publicáronse revistas y periódicos de importancia [...] por efímeras y modestas que fuesen tales publicaciones, indicaban un interés [...] por ALGO TAN IMPORTANTE PARA CUALQUIER CRECIMIENTO LITERARIO COMO LA DIVERSA EDICIÓN DE PERIÓDICOS Y REVISTAS (Saturno 74-75). [Durante este período, PUBLICÁRONSE REVISTAS Y PERIÓDICOS de importancia. Por efímeras y modestas que fuesen tales PUBLICACIONES, indicaban un interés por ALGO TAN IMPORTANTE PARA CUALQUIER CRECIMIENTO LITERARIO]

También puede suprimirse el término si es de conocimiento general $-\mathrm{o}$ al menos del oyente específico a que se dirige el mensaje-, y la sola mención del núcleo consigue referirlo inequívocamente, como sucede en la siguiente secuencia:

11) Yo envidio al príncipe de Gales por su palacio de Buckingham y porque fue capaz de abandonar a UNA PRINCESA COMO DIANA (Cándido 73). [Yo envidio al príncipe de Gales por su palacio de Buckingham y porque fue capaz de abandonar a UNA PRINCESA]

\section{VALORES SEMÁNTICOS Y SINTÁCTICOS DE COMO EN LAS SECUENCIAS PONDERADORAS DE IDENTIFICACIÓN}

El carácter ponderativo de estas secuencias descansa en el valor cuantitativomodal de como y en el orden de los constituyentes. El valor semántico modal de como se manifiesta a través del correlato anafórico que establece con una cualidad previamente expresada, mientras que su referencia cuantitativa se vincula, básicamente, con la intensificación a que puede someterse tal cualidad.

Pero la función de estas secuencias no es solo la ponderación, sino también la identificación referencial de los sintagmas básicos que la constituyen. Y la identificación se consigue a través de la resemantización de la estructura comparativa de igualdad tan...como, haciendo que el adverbio intensificador tan, en vez de igualar el grado en que se manifiesta la cualidad en el sustantivo encabezado por como respecto del sustantivo anterior, exprese el más alto grado de manifestación de la cualidad como propio del sustantivo introducido por como, para que la cualidad se convierta en atributo de identificación de este. Utilizando la estructura binaria típica de la comparación de igualdad la significación del adjetivo resulta, no obstante, la del superlativo absoluto.

12) Cuando el Che muere en Bolivia en 1967 habían transcurrido tan solo cuatro años de la publicación DE UN VOLUMEN TAN VIOLENTAMENTE PERSONAL COMO LIBRO DE LOS EPÍLOGOS (Presunciones 189).

NORMAS. REVISTA DE ESTUDIOS LINGÜÍSTICOS HISPÁNICOS, NÚMERO 3 (AÑO 2013):

http://www.uv.es/normas

(ISSN 2174-7245) 
Esta apropiación formal de la estructura comparativa propicia que en las secuencias ponderadoras de identificación -a semejanza de las construcciones propiamente comparativas- pueda no expresarse el intensivo tan, y sin embargo, su significación quede «presupuesta en el reconocimiento del como», puesto que tan y como «se hallan en una correlación biunívoca» (Gutiérrez, 2000: 3) -aunque no sea esta la única circunstancia que puede favorecer la omisión del intensificador, según se verá más adelante- .

Otro tanto sucede con la cualidad intensificada, que en muchas ocasiones no se explicita. Para Gutiérrez Ordóñez (2000: 8), la existencia de la cualidad «queda sugerida por la significación del como, que se comporta como un operador procedimental [...] es decir, como un signo que aporta fundamentalmente unas instrucciones de descodificación». Se produce entonces un caso típico de comunicación ostensivoinferencial porque «la capacidad inferencial de los hablantes, apoyada en el soporte de algunos datos formales, semánticos y contextuales, suple lo que falta [explícitamente en el mensaje]»(Gutiérrez, 2000: 4), parecido a lo que sucede con las comparaciones prototípicas:

13) Edmundo Bastidas dijo sentirse feliz porque su hermano descanse eternamente en UN PAÍ́ COMO CUBA (Granma, mayo 14: 2).

El hecho de que la calificación -explícita o implícita- a que hace referencia anafórica la partícula debe estar sometida -explícita o implícitamente- a graduación (cuantificación intensiva), explica que en casos como el siguiente la referencia anafórica de como debe seguir considerándose presupuesta, porque el adyacente - un adjetivo relacional- no resulta graduable en el contexto:

14) En UN CUERPO [*TAN] LITERARIO COMO EL DE CUBA, que comenzó a crecer bajo el reinado de la imagen hace 400 años con el poema épico Espejo de paciencia, de Silvestre de Balboa, no es sorprendente que varios creadores se hayan expresado indistintamente desde la palabra y el lienzo (Granma, mayo 23: 13).

En resumen: la partícula como, gracias a su significación cuantitativo-modal, puede funcionar en calidad de operador procedimental que induce la interpretación de una cualidad intensificada que no aparece explícita.

Pero para la descripción de las secuencias ponderadoras de identificación no basta con la comprensión del valor semántico de como; se hace necesario, también, analizar su comportamiento sintáctico, tema sumamente controvertido.

A favor de la condición de adverbio relativo está el hecho inobjetable de su significación cuantitativo-modal y de la referencia anafórica que mantiene. Sin 
embargo, la ausencia sistemática del verbo en estas secuencias hace muy dudoso postular su elipsis, y sin una estructura verbal donde como pueda desempeñar una función sintáctica oracional, pierde su capacidad de sustituir las unidades con las cuales establece el correlato fórico.

El carácter no oracional y subordinado del sintagma introducido por la partícula, así como la incapacidad sustitutoria de esta podrían hacer pensar que como se desempeña en calidad de conjunción subordinante (específicamente comparativa) o preposición. En uno y otro caso, no obstante, queda una contradicción sin resolver: el hecho de que, a pesar de todo, como sigue teniendo función fórica.

Por otra parte, la postulación de este como conjuntivo arrastra el dilema -aún sin solución- de la existencia o no de conjunciones verdaderamente subordinantes (Montoro, 2005: 417-423) ${ }^{6}$, así como las dificultades para determinar la naturaleza de los nexos comparativos, que comparten rasgos de las conjunciones coordinantes y subordinantes (RAE $\S 31.1 .3$ ). Sin embargo, en dependencia de que el presunto verbo elíptico pueda reponerse o no en el decurso, es común adjudicarle a como, respectivamente, carácter relativo o conjuntivo comparativo (RAE, 2010: 422).

El criterio de la reposición del verbo para deslindar entre el uso adverbial relativo y conjuntivo comparativo no puede aplicarse coherentemente al análisis de las secuencias específicas que se estudian aquí, porque es posible en unos casos y en otros no:

15) Lo más importante se centra hoy en completar la contratación de toda la campaña, en aras de mejorar los resultados y dignificar UN RUBRO EXPORTABLE TAN SIGNIFICATIVO COMO EL TABACO (Escambray, junio 28: 8). [Lo más importante se centra hoy en completar la contratación de toda la campaña, en aras de mejorar los resultados y dignificar UN RUBRO EXPORTABLE TAN SIGNIFICATIVO COMO ES EL TABACO]

${ }^{6}$ A partir de la tríada de funciones sintácticas propuesta por Hjelmslev, 1971 (determinación, interdependencia y constelación), algunos funcionalistas que estudian las llamadas oración compuesta (coordinadas e interordinadas) y oración compleja (subordinadas), impugnan la existencia de conjunciones de subordinación. Según estos autores, las conjunciones son elementos de relación que conectan unidades con el mismo rango sintáctico y en esa medida solo pueden realizar las funciones de constelación e interdependencia. La función de determinación, propia de la subordinación -unidades de distinto rango funcional en que una se comporta como núcleo y la otra como adyacente-, solo es posible a través de la preposición y de los llamados inclusores, dentro de los cuales se halla, por ejemplo, que anunciativo, tradicionalmente entendido como conjunción subordinante. Sostienen estos análisis que los auténticos elementos de relación (conjunciones y preposiciones) pueden conectar unidades dentro del sintagma, mientras que a los inclusores les está vedada tal posibilidad, toda vez que su ámbito de actuación es exclusivamente la oración compleja, en la cual habilitan una unidad predicativa (las llamadas tradicionalmente proposiciones) para incluirse dentro de otra unidad predicativa (la oración regente).

NORMAS. REVISTA DE ESTUDIOS LINGÜÍSTICOS HISPÁNICOS, NÚMERO 3 (AÑO 2013):

http://www.uv.es/normas

(ISSN 2174-7245) 
16) La foto de Phillips es un artefacto con sabor a burdel caro [...] pero ahí está la trama subterránea de UN RELATO COMO «CUATRO LIBRAS DE FELICIDAD», o lo que nos oculta la sinuosa conducta de los personajes en «El gato», o lo que sucede tras el desenlace de «La puerta falsa», o el tema del sexo en «El drama de la señorita Occidente» (Presunciones 90). [*Pero ahí está la trama subterránea de UN RELATO COMO ES «CUATRO LIBRAS DE FELICIDAD», o lo que nos oculta...]

Solo queda entonces la posibilidad de considerar un como prepositivo que subordina un segmento no oracional a un elemento previo del discurso, y se desentiende, por tanto, de la hipótesis sobre el verbo elíptico.

Aunque la interpretación prepositiva de como deja sin resolver la cuestión de la foresis cuantitativo-modal de la partícula y debe enfrentarse al hecho de que como en estas secuencias no cumple con requisitos propios de la preposición como la posibilidad de regir formas complementarias de pronombres personales, no resulta extraña la adjudicación del valor sintáctico prepositivo a una partícula de ascendencia adverbial relativa, considerando las investigaciones sobre la imbricación entre adverbio, adverbio con función preposicional y preposición en el pensamiento lingüístico hispánico y de otras lenguas (Cifuentes, 1989 : 266-281).

Así, más que dirimir -al modo de la RAE- si el presunto verbo puede reponerse o no, y derivar de ello el comportamiento adverbial relativo o conjuntivo de como, resulta más objetivo invertir la perspectiva de análisis y comprobar si el verbo aparece o no, y en caso de estar, si puede suprimirse o no. En solo tres realizaciones de la muestra el copulativo aparece:

17) A pesar de estar prestando servicios ambos parques [...] y del esfuerzo de muchos compañeros, la inversión no ha concluido pues faltan temas como la telefonía, equipamiento gastronómico, y UN ASUNTO BIEN COMPLEJO COMO ES EL ABASTO DE AGUA [...] (Granma, mayo 23: 11).

Este hecho concuerda con las observaciones de la mayoría de los gramáticos, especialmente con las de Cano (1995: 54), quien reconoce la ausencia sistemática del verbo en estas y otras construcciones análogas de como desde los orígenes del idioma. Cabe entonces suscribir la idea de Tomás Jiménez Juliá -referida a otras realizaciones de como- de que «la construcción sin verbo está reflejando una estructura preposicional que la lengua ha aceptado como tal, sin necesidad de descartar la existencia paralela de la relativa con verbo, como construcción distinta, aunque similar referencialmente» (Jiménez Juliá, 2003: 25).

Nótese como estas secuencias con verbo pueden rescribirse sin él:

Faltan temas como la telefonía, equipamiento gastronómico, y UN ASUNTO BIEN COMPLEJO COMO EL ABASTO DE AGUA. 
Por consiguiente, consideraremos -al menos desde un punto de vista operativo- que en las secuencias ponderadoras de identificación de como $\sin$ verbo $^{7}$ la partícula ha transformado su función de relativo (subordinante oracional) en mera relación de determinación entre sintagmas, convirtiendo el segmento que introduce en adyacente nominal del precedente, similar al comportamiento preposicional en los grupos nominales seudoapositivos del tipo $N$ de $N$, en los cuales es evidente la correferencia de los elementos relacionados por la preposición:

18) El asunto de metamorfosear al pícaro clásico y situarlo, en movimiento, dentro de UNA CIUDAD COMO LA HABANA A FINES DEL SIGLO XX, ha puesto a Jorge Ángel Pérez en una estimulante situación de riesgo literario (Presunciones 352). [El asunto de metamorfosear al pícaro clásico y situarlo, en movimiento, dentro de LA CIUDAD DE LA HABANA A FINES DEL SIGLO XX, ha puesto a Jorge Ángel Pérez en una estimulante situación de riesgo literario]

El enfoque prepositivo de como permitirá, a los efectos de la descripción morfosintáctica de estas construcciones, denominar «núcleo» de la secuencia al sustantivo (o unidad equivalente) que encabeza el primero de los sintagmas de la secuencia y llamar «término» de la secuencia a la(s) unidad(es) introducidas por como en el segundo de los sintagmas. Sin embargo, es importante considerar que el «término» no es solo adyacente del sustantivo (o unidad equivalente) nombrado «núcleo», sino que se subordina al conjunto de los miembros que constituyen el primer sintagma, y que llamaremos «sintagma nuclear».

El escollo más notable para esta interpretación sigue siendo -como se ha dicho- la foresis cuantitativo-modal de como, difícilmente compatible con las preposiciones. Solo cabe pensar que tan paradójico comportamiento de la partícula responde a los procesos de gramaticalización a que se ha visto sometida en su condición de palabra estructural o funcional. Resumiendo las ideas de Heine, Claudi y Hünnemeyer (1991) sobre estos procesos, Cifuentes Honrubia (2002: 45) afirma:

[...] la gramaticalización es un continuo [...] una transición gradual [...] Y en ese continuo podemos hablar de un estado intermedio en el que la entidad no puede ser descrita como siendo fundamentalmente $\mathrm{X}$ ni fundamentalmente $\mathrm{Y}$, ya que tiene una cantidad proporcional de ambas categorías. Las formas híbridas son, por tanto, parte de las cadenas de gramaticalización.

7 Obviamente, en las secuencias ponderadoras de identificación donde aparece el verbo, la partícula como encabeza una oración de relativo.

NORMAS. REVISTA DE ESTUdIOS LINGÜÍSTICOS HISPÁNICOS, NÚMERO 3 (AÑO 2013):

http://www.uv.es/normas

(ISSN 2174-7245) 
Basándonos en esta idea sobre los procesos de gramaticalización, estimamos pertinente postular la forma híbrida de como en las construcciones analizadas: de la categoría adverbial preserva el carácter cuantitativo-modal de su significación; de la preposición adquiere la capacidad de introducir sintagmas subordinándolos como adyacentes nominales.

\section{NÚCLEO DE LAS SECUENCIAS PONDERADORAS DE IDENTIFICACIÓN CON COMO}

Las secuencias ponderadoras de identificación con como más típicas suelen tener por núcleo un sustantivo común en singular, actualizado por el indefinido un/una:

19) $[\ldots]$ Es [...] perceptible en uno de sus grandes poemas, «West Indies Ltd.», y también En UN POEMARIO TAN PROFUNDAMENTE EMOCIONAL Y DINÁMICO COMO «EL SOLDADO MigUEL PAZ Y EL SARGENTO JOSÉ INÉS» (Saturno 186).

En la muestra también se hallan secuencias con el indefinido algo en función nuclear:

20) El gran triunfo de esa inoculación ha consistido siempre en mover, o conmover la conciencia del individuo. Sacudirla con ALGO TAN QUEBRADIZO Y CORPULENTO COMO EL LENGUAJE DE LA LITERATURA (Presunciones 415).

Es posible, además, encontrar algunas con núcleo compuesto por un sustantivo en plural, actualizado por el numeral cardinal dos:

21) [...] volvamos al asunto del principio: la República como un ajustado profile de barbarie natural, desorden, provincianismo e intemperancia en DOS NOVELISTAS COMO BOBADILLA Y LOVEIRA (Presunciones 84).

Aunque las anteriores realizaciones de como se ajustan de manera más inobjetable a la correferencia estricta que define a las secuencias ponderadoras de identificación, el grueso de la muestra está constituido por secuencias cuyo sustantivo núcleo en plural se halla inactualizado léxicamente (22). En otras, aparece determinado por indefinidos de la serie otro/a (23), y en unas pocas, el propio indefinido cumple la función nuclear en la secuencia (24):

22) Y si escribo la palabra sabor, debo escribir, además, PALABRAS COMO TEXTURA, RELIEVE E, INCLUSO, COLOR Y AUDIBILIDAD [...] (Presunciones 326).

NORMAS. REVISTA DE ESTUDIOS LINGÜÍSTICOS HISPÁNICOS, NÚMERO 3 (AÑO 2013):

http://www.uv.es/normas

(ISSN 2174-7245) 
23) Es visible una complicidad con OTRAS CARAS DE LA INDUSTRIA CULTURAL COMO EL CINE Y EL SECTOR PUBLICITARIO, donde la supuesta libertad de creación en el mundo virtual es presa de desgastadas fórmulas de consumo (Granma, junio 6: 7).

24) El programa consta de 15 deportes, algunos locales, pero OTROS TAN UNIVERSALES COMO ATLETISMO, NATACIÓN Y FUTSAL (Granma, junio 6: 15).

Como ha podido observarse, cuando el núcleo aparece en singular, la presencia de un determinante que actualice su referencia es obligatoria -salvo que se trate de un indefinido como algo, cuantificador actualizado implícitamente-:

25) [...] la conexión de Yulieski [...] le pasó por encima a UN JUGADOR DE BUENA ESTATURA COMO EL CAMARERO RAFAEL VALDÉS y se internó de jit en el bosque derecho [...] (Granma, abril 14: 7). [La conexión de Yulieski le pasó por encima a *JUGADOR DE BUENA ESTATURA COMO EL CAMARERO RAFAEL VALDÉS y se internó de jit en el bosque derecho]

Por el contrario, las realizaciones con núcleo en plural parecen no requerir forzosamente la actualización léxica, quizá porque «el morfema de plural intervendría como "actualizador" (una elemental cuantificación) y, por tanto, concedería al sustantivo una cierta capacidad denotadora» (Martínez, 1994b: 190-191) ${ }^{8}$ :

26) [...] un acuerdo adoptado por el Consejo de Ministros en octubre de 2007 indica que ORGANISMOS COMO EL SIME, MINBAS, MINIL, MINAZ, MICONS, MINAL, MINAGRI Y MINCIN laboran para garantizar la entrega de los productos en las provincias donde radican los consumidores finales en incluso, donde sea posible, en los municipios (Escambray, abril 5: 8).

Sin embargo, es preciso destacar que en las secuencias de núcleo en plural si bien la presencia del actualizador numeral contribuye a remarcar la correferencia estricta entre este y el término, en los restantes casos se advierte una ambigüedad referencial que puede acercar estas realizaciones a los usos adscriptivo o ejemplificador de como ${ }^{9}$.

${ }^{8}$ En sus Funciones, categorías y transposición, Martínez (1994a:107) sostiene la misma idea: «[...] el término marcado del morfema de número, el plural, representa una suerte de «cuantificación» mínima, por lo que puede ser considerado como "actualizador"».

9 Aunque un análisis de tal naturaleza rebasa el interés y alcance de este trabajo, pensamos que la presencia/ausencia del intensificador tan así como de los adyacentes del núcleo en el primer sintagma de la secuencia favorece una u otra interpretación. Así, se hará más plausible la interpretación adscriptiva cuando aparece el intensificador o cuando, por el contrario, el núcleo carece de estos adyacentes. En cambio, la presencia de ellos en ausencia de tan favorece la interpretación ejemplificadora, toda vez que

NORMAS. REVISTA DE ESTUDIOS LINGÜÍSTICOS HISPÁNICOS, NÚMERO 3 (AÑO 2013):

http://www.uv.es/normas

(ISSN 2174-7245) 
Por otra parte, cabe sostener que el valor identificador de las secuencias con como se relaciona íntimamente con la actualización no identificadora que afecta al núcleo, sea a través de los determinantes (actualizadores léxicos), del morfema de plural o de la naturaleza indefinida del propio núcleo cuando algo u otro/a(s) desempeñan esa función.

Prueba de que el actualizador del núcleo -tanto en singular como en plural- debe, forzosamente, excluir el valor identificador del artículo, es que la inclusión de este hace agramatical la mayoría de los decursos ${ }^{10}$ :

27) La muchachita [...] dejó a casi tres segundos A UNA RIVAL DE ALCURNIA COMO MARYAM JAMAL (Granma, mayo 26: 7). [La muchachita dejó a casi tres segundos a *LA RIVAL DE ALCURNIA COMO MARYAM JAMAL]

solo en estos casos es posible independizar entonativamente el segmento encabezado por como (v. nota 10). Tanto en una situación como la otra, sin embargo, consideramos que estas realizaciones son básicamente ponderadoras de identificación (v. supra § «Definición y criterios de exclusión»), aunque lo sean menos típicamente que las de núcleo en singular o las de núcleo en plural actualizado por un numeral cardinal descritas aquí. Apoya este criterio el hecho de que, por una parte, las secuencias de como con interpretación firmemente adscriptiva parecen darse en contextos genéricos o en presencia de modalizadores, y por otra, que en las construcciones ejemplificadoras típicas el sintagma encabezado por como es una unidad parentética. Esto último es constatable en Cuervo (1998:221,232), Alcina y Blecua (1994: 1080), Cano Aguilar (1995:64) y Jiménez Juliá (2003:32). En la bibliografía consultada, solo Felisa Sanjuán (1997:34) y la RAE (2010: § 45.3.1f) describen el valor ejemplificativo de como en construcciones no parentéticas.

De todas formas, para corroborar estas ideas habrá que repensar y contraponer los conceptos de adscripción y ejemplificación así como correlacionarlos con la identificación y las diversas posibilidades interpretativas que ofrece la referencia (específica, exclusiva, prototípica) de los enunciados donde se insertan secuencias de como afines a estas que se analizan.

${ }^{10}$ En algunas realizaciones donde el término es una enumeración y el núcleo en plural presenta adyacentes en ausencia del intensificador es posible eliminar la agramaticalidad del decurso resultante tras la inclusión del artículo. Basta con independizar entonativamente el término de la secuencia. Este cambio, sin embargo, hace que la secuencia pierda sus valores de ponderación e identificación y se altere el sentido del enunciado: «Me refiero a textos aparecidos en PUBLICACIONES PERIÓDICAS COMO CHIC, SOCIAL, LA PRENSA, CARTELES Y BOHEMIA» (Presunciones 75). [= aparecidos únicamente en las publicaciones periódicas Chic, Social, La Prensa, Carteles y Bohemia]. «Me refiero a textos aparecidos en las publicaciones periódicas, como ChiC, Social, LA PRENSA, CARTELES Y BOHEMia». [= aparecidos en (¿todas o cualesquiera?) las publicaciones periódicas, entre ellas Chic, Social, La Prensa, Carteles y Bohemia]

Como puede advertirse, el sintagma introducido por como adquiere valor de ejemplificación, con el claro matiz extrapredicativo de la función incidental. El término constituye un mero refuerzo concretizador del núcleo. Nótese que la secuencia original puede rescribirse de igual forma y adquiere idéntico valor ejemplificador: «Me refiero a textos aparecidos EN PUBLICACIONES PERIÓDICAS, COMO CHIC, SOCIAL, LA PRENSA, CARTELES Y BOHEMIA». 
28) Ha sido bonito, me ayudaron mucho DOS PROFESORAS COMO MARÍA ISABEL CASTAÑEDA Y CARMEN VARONA, y ahora todo el colectivo (Escambray, mayo 24: 7). [Me ayudaron mucho *LAS dOS PROFESORAS COMO MARÍA ISABEL CASTAÑEDA Y CARMEN VARONA]

Además de los actualizadores o determinantes y del término de la secuencia, el núcleo puede presentar otros adyacentes, calificativos y no calificativos. La ponderación de la secuencia, obviamente, descansa en los adyacentes calificativos, salvo que los no calificativos sean reinterpretados como calificativos en el contexto:

29) Lezama [...] no se interesó por conceptualizar la pervivencia barroca en las artes de América en el siglo XX. Sí percibió el fenómeno, como se evidencia en sus consideraciones sobre el estilo barroco en UN PINTOR CUBANO [TAN] CONTEMPORÁNEO COMO MARIANO, pero no ahondó en esta cuestión (Saturno 207).

Aunque la calificación se expresa en adyacencia al núcleo, afecta tanto al núcleo como al término, por dos razones: desde el punto de vista sintáctico, el término es adyacente del sintagma nuclear (compuesto por el determinante o actualizador, el núcleo y el resto de los modificadores -calificativos y no calificativos- que preceden a como); y desde el punto de vista semántico, en virtud de la identificación referencial que establece la secuencia entre el núcleo (o sintagma nuclear) y el término, la cualidad es atribuida, en última instancia, como una cualidad inherente al término.

En las secuencias ponderadoras de identificación de la muestra la calificación aparece intensificada, frecuentemente, por la presencia del adverbio tan, que de esta manera -superlativo absoluto- expresa el más alto grado de manifestación de la cualidad como propio del término:

30) $[\ldots]$ en direcciones semejantes [...] se moverán POETAS TAN RELEVANTES COMO PovedA, Boti y AGUSTín ACOSTA (Saturno 99).

Algunas veces la cualidad no aparece intensificada por tan sino por un adverbio afín:

31) GUANTÁNAMO. PARA UNA PROVINCIA EMINENTEMENTE AGRÍCOLA COMO ESTA [...] fue de mucho acierto que los asistentes a la Asamblea Provincial del Partido centraran sus debates en el tema de la producción de alimentos (Granma, junio 16: 3).

NORMAS. REVISTA DE ESTUDIOS LINGÜÍSTICOS HISPÁNICOS, NÚMERO 3 (AÑO 2013):

http://www.uv.es/normas

(ISSN 2174-7245) 
También puede expresarse la cualidad como inherente o consustancial al sustantivo -circunstancia que hace redundante la presencia del intensificador tan- a través de otros medios:

a) Anteposición del adjetivo calificativo respecto del núcleo:

32) «Nos enfrentamos a UN GRAN EQUIPO COMO LA HABANA, nos tocó a nosotros esta vez; los muchachos se crecieron, el pitcheo de Ismel se comportó a la altura que esperábamos, todo el equipo de comportó bien [...]» (Escambray, abril 5: 7).

b) Calificación a través de elativos léxicos (RAE § 13.2.3):

33) Para Un PAís INMENSO COMO RUSIA, Europa Occidental no es solo un centro de cultura, arte, historia y ciencia refinada $[\ldots]$ es también consumidora de petróleo y gas, oro, níquel y materias primas rusas, instrumento para la fuga del capital y cerebros [...] (Granma, abril 7: 4).

c) Calificación a través de otros adjetivos (o complementos preposicionales de interpretación calificativa o estimativa) de significación cercana a la de los elativos léxicos:

34) $[\ldots]$ por entonces ya había escrito TEXTOS DE IMPRESIONANTE SAZÓN LINGUOESTILÍSTICA COMO «AQUELLA NOCHE SALIERON LOS MUERTOS», «EN EL CAYO» Y «LA LUNA DE LOS ÑÁNIIGOS» (todos en 1932 y aparecidos en la Revista de Occidente) (Presunciones 162).

La calificación puede no aparecer de manera explícita. En tales casos -que son mayoritarios en la muestra- habrá de presuponerse, en virtud de la referencia cuantitativo-modal de como y el valor ponderador de la secuencia:

35) Amir Valle escribió UN TEXTO COMO «MAMBRÚ NO FUE A LA GUERRA», una historia apegada a lo real, a las crueldades del destino, y que nos cuenta cómo un joven $[\ldots]$ (Presunciones 277).

36) Realizó [Calvert Casey] una exploración audaz del entorno, específicamente de FENÓMENOS COMO LA PROSTITUCIÓN Y LA VIOLENCIA (Presunciones 68).

Esta presuposición no implica que la ausencia del adjetivo o expresión calificativa equivalente deba interpretarse como elipsis. Se trata de casos evidentes de no 
realización $^{11}$ que obedecen a mecanismos típicos de la comunicación ostensivoinferencial.

\section{TÉRMINO DE LAS SECUENCIAS PONDERADORAS DE IDENTIFICACIÓN CON COMO}

El término de las secuencias ponderadoras de identificación con como suele presentar características morfosintácticas muy diversas. En primer lugar, pueden constituirlo una o varias unidades, a las cuales les está permitido:

a) ser sustantivos propios, sustantivos comunes, pronombres en función sustantiva $\mathrm{u}$ otras unidades sustantivadas por la anteposición del artículo $^{12}$ : grupos prepositivos, oraciones de relativo, adjetivos y posesivos;

b) presentarse en singular o plural;

c) estar actualizados -explícita o implícitamente- con el valor identificador del artículo o aparecer inactualizados tanto léxica como morfemáticamente.

Esta variedad de comportamiento, sin embargo, se halla sujeta a ciertas restricciones. Entre el término y el núcleo debe existir concordancia de número, que puede darse morfemáticamente, tanto en singular como en plural:

37) Bill Clinton [...] puede asesorarlo en UN TEMA COMO EL BLOQUEO, que prometió erradicar y nunca cumplió (Granma, mayo 26: 2).

38) Se distinguen por el sello de calidad PRODUCTOS COMO LOS ÁRIDOS DE LA CANTERA NIEVES MOREJÓN, reconocidos en el 2002 con el Premio Nacional de la Calidad que otorga anualmente el Ministerio de la Construcción de Cuba (Escambray, junio 28: 3).

${ }^{11}$ Para una distinción entre elipsis, elisión y no realización, véase Juliá (1991).

${ }^{12}$ En esta cuestión polémica sobre la función sustantivadora del artículo se sigue el criterio de Alarcos Llorach (1984: 229): «[...] cuando un artículo se antepone a elementos cuya función habitual no es la de nombre, el papel de aquel evidentemente consiste en trasponer tales elementos a la función que el nombre desempeña en la oración. Sin duda, pues, el artículo es un traspositor a nombre de cualquier otro elemento funcional: el blanco, la antigua [...] los que viven, el de la derecha [...]». Como puede notarse en el tercero de los ejemplos en cursiva, para las relativas con artículo antepuesto, Alarcos -al igual que Gili y Gaya (1975 § 231)- considera válido el mismo razonamiento, que desarrolla así: «Si las transposiciones con /que/ 2 funcionan como adjetivos, podrán también, como estos, ser de nuevo transpuestas de la función adyacente a la función nuclear del grupo sintagmático nominal. En este caso, el elemento transpositor es el mismo que con los adjetivos, el "artículo"» (Alarcos, 1984: 265).

NORMAS. REVISTA DE ESTUDIOS LINGÜÍSTICOS HISPÁNICOS, NÚMERO 3 (AÑO 2013):

http://www.uv.es/normas

(ISSN 2174-7245) 
Pero en la mayoría de las secuencias de núcleo en plural, el término se halla integrado por varios sustantivos (en singular y/o plural) coordinados copulativamente, y la concordancia se establece a través del plural sintáctico:

39) Estamos muy preocupados con el afloramiento de DOS FENÓMENOS O EXPRESIONES CRIMINALES COMO EL SECUESTRO Y LAS AMENAZAS (Granma, mayo 22: 8).

En cuanto a la categoría de género, el comportamiento de la concordancia entre el núcleo y el término es mucho más complejo. Se establece rigurosamente solo cuando el término es un pronombre $\mathrm{u}$ otra unidad sustantivada por la anteposición del artículo:

40) $[\ldots]$ decidieron abrir el féretro para limpiar el cristal [...] Justamente lo que yo quería. Antes me enteré de que UNA OPERACIÓN COMO ESTA no pondría en peligro el trabajo del embalsamador (Fumando 222).

41) Cuando la conversación se desliza, fluye, dijo Jenofonte [...] de pronto un personaje $[\ldots]$ la interrumpe $[\ldots]$ no solo quiere convencernos, sino $[\ldots]$ convencerse de cuanto dice [...] Nunca será un conversador este tipo [...] UN TIPO COMO EL DESCRITO POR JENOFONTE me parece un hombre inseguro en grado superior al resto de los mortales (Noche 31-32).

Pero -similar a lo que sucede en las oraciones copulativas adscriptivas entre el núcleo del sujeto y el sustantivo en función atributo- las marcas formales de concordancia desaparecen en el resto de los casos, que son la mayoría:

42) UN FENÓMENO COMO LA ESCRITURA SORPRENDIDA, la escritura que se hace antes de 1959 y se da a conocer después de esa fecha, es siempre de interés (Presunciones 188).

Si el término está compuesto por nombre(s) propio(s) o expresión(es) denominativa(s), la concordancia, tanto de número como de género, se comporta igual que en otros contextos de la lengua española:

43) Ness [...] tiene en la protección de su familia un protésico, extirpable y lejano atributo de masculinidad [...] que encarna en UN ACTOR COMO KEVIN COSTNER, cuya expresión de pobre-buen-hombre viene como anillo al dedo del personaje (Hombre 111).

44) [...] la medida podría extenderse a CIUDAdES COMO SAN FrANCISCO Y CHICAgo (Granma, junio 6: 8). 
45) Ya se había publicado UNA NOVELA TAN ESCOLTADA Y BIEN HECHA COMO EL PAN DORMIDO, pero a mí no acababa de gustarme (Presunciones 196).

Como se verá, para el término que concuerda en singular con el núcleo es obligada la actualización -explícita o implícita- con el valor identificador del artículo (46-50, 52 y 53). Cuando el término de como concuerda con el núcleo en plural, esta actualización, de forma general, se mantiene (51), aunque en algunos casos puntuales no se observa o lo hace de manera especial (54 y 55).

A continuación aparecen ejemplos de la muestra que ilustran las diferentes unidades que pueden constituir el término, así como el comportamiento de su actualización:

a) Sustantivos comunes actualizados explícitamente por el artículo:

46) En este retorno a la primera persona de la cercana voz autoral, en UN MOMENTO TAN METADISCURSIVO COMO LA PRESENTACIÓN, se retoma la idea de pluralidad y del fragmento (Diálogos 72-73).

b) Una unidad sustantivada por la anteposición del artículo, la cual puede ser:

- Un grupo prepositivo:

47) El dilema estético de UNA LiTERATURA COMO LA DE COLlazo estriba en las sucesivas metamorfosis que detentan su prosa y los asuntos asediados en sus libros (Presunciones 225).

- Un adjetivo:

48) [...] las dos alternativas esenciales del escritor de ficciones en UNA REALIDAD COMO LA CUBANA se hallaban dominadas por la vertiginosa existencia inmediata y por una imaginación que trascendía esa inmediatez [...] (Presunciones 276-277).

- Un posesivo:

49) Pero este viejo ardid de los culebrones [...] se ha ido complejizando hasta el punto de integrarse sin remilgos a la llamada cultura de masas [...] al margen de estas consideraciones pudiera abrirse un paréntesis con las telenovelas cubanas que prescinden de estos condicionantes extinguidos en UNA SOCIEDAD COMO LA NUESTRA y enseguida salta a la vista otra de las funciones de la comunicación social [...] (Escambray, junio 28:0).

NORMAS. REVISTA DE ESTUDIOS LINGÜÍSTICOS HISPÁNICOS, NÚMERO 3 (AÑO 2013):

http://www.uv.es/normas

(ISSN 2174-7245) 
- Una oración de relativo con que:

50) A principios de los años 20 el ensayista Jorge Mañach dio a conocer un relato de cierta extensión titulado Belén el aschanti [...] el Belén de Mañach empieza a ser un personaje para el contraste y su ir y venir por UN RELATO COMO EL QUE LE DA VIDA no cayó en el vacío (Presunciones 123-124).

c) Sustantivo(s) propio(s) actualizado(s) implícitamente:

51) [...] quedó instalada la exposición Homenajeando, con obras de CONOCIDOS artistas COMO Nelson Domínguez, Lesbia Vent Dumois, ERnesto García Peña, Adigio benítez, Tato Ayress, José Omar, Virginia Ayress, Carlos VERdiAl, MOISÉS FinAlÉ Y CARLOS DEL TORO (Granma, junio 13: 12).

d) Forma tónica de los pronombres personales actualizada implícitamente:

52) Ya sé $[\ldots]$ que sus versos [...] no están hechos para fines lucrativos, pero siéntase contento, feliz, de que a UNA VENDEDORA DE TORTILLAS COMO YO le sirvieran para algo (Fumando 41).

e) Demostrativo en función sustantiva actualizado implícitamente:

53) Entonces, camarero, que tan portentosa bebida cierre esta noche espléndida y nos purifique. Póngalo en mi cuenta. En UNA NOCHE COMO ESTA me siento generoso (Noche $50)$.

f) Sustantivos comunes inactualizados léxicamente, los cuales -aunque muy escasos- exhiben comportamientos diferentes. Pueden ser:

- Sustantivos comunes actualizados morfemáticamente por la marca de plural:

54) El empeño [...] se diversifica al ensanchar su horizonte constructivo hasta la fundición de casas aisladas del sistema antiguo, pedidos para obras de la Batalla de Ideas, grupos electrógenos y OTROS ELEMENTOS ALTERNATIVOS COMO BATEAS, LOSAS, TECHOS, VIGAS Y TAPAS DE REGISTROS (Escambray, junio 28: 5).

- Sustantivos comunes que aparecen inactualizados léxica y morfemáticamente (están en singular), pero cuyo valor denominativo y la noción de «unicidad» (RAE $\S 12.5 .2 \mathrm{~d}, 12.7 .4 \mathrm{c})$ que expresan, hacen asimilable su significación a los nombres propios no personales, de actualización identificadora implícita:

NORMAS. REVISTA DE ESTUDIOS LINGÜÍSTICOS HISPÁNICOS, NÚMERO 3 (AÑO 2013):

http://www.uv.es/normas

(ISSN 2174-7245) 
55) En cuanto a coach [...] su uso es innecesario en español, pues existen TÉRMINOS COMO ENTRENADOR Y PREPARADOR, de sentido equivalente (Granma, mayo 27: 2).

\section{CONCLUSIONES}

Las secuencias ponderadoras de identificación con como resultan una construcción lingüística peculiar, de orden invariable, integrada por dos sintagmas nominales, el primero de los cuales -el núcleo- denota la clase a que pertenece el segundo -el término-, ligado entonativamente con aquel e introducido por la partícula como. La extensión referencial del núcleo se hace mínima en el contexto para identificarse o igualarse con la del término, de manera que se establece entre ambos una correferencia estricta y se encarece o pondera una cualidad -positiva o negativa, implícita o explícita- de la clase denotada por el núcleo, expresándola como inherente al término.

En las secuencias ponderadoras de identificación donde no aparece verbo alguno tras como, es plausible postular el estatus híbrido de la partícula: si bien conserva rasgos semánticos propios de la categoría adverbial -hecho notorio en la significación cuantitativo-modal de su foresis-, desde el punto de vista estrictamente sintáctico, ha transformado su función de relativo o subordinante oracional en mera relación de determinación entre sintagmas, convirtiendo el segmento que introduce en adyacente nominal del precedente, similar al comportamiento prepositivo.

De manera general, puede concluirse que las unidades que integran las secuencias ponderadoras de identificación con como son las siguientes:

\begin{tabular}{|c|c|c|c|}
\hline \multicolumn{2}{|r|}{ SINTAGMA NUCLEAR } & \multicolumn{2}{|c|}{ SINTAGMA ADYACENTE } \\
\hline $\begin{array}{l}\text { Núc } \\
\text { leo }\end{array}$ & $\begin{array}{l}\text { Adyacentes calificativos y no } \\
\text { calificativos }\end{array}$ & $\begin{array}{l}\mathrm{CO} \\
\mathrm{MO}\end{array}$ & Término \\
\hline \multirow[t]{2}{*}{+} & $+1-$ & \multirow{2}{*}{+} & \multirow{2}{*}{+} \\
\hline & $\begin{array}{l}\text { Intensificador tan } \mathrm{O} \\
\text { afines } \\
+/-\end{array}$ & & \\
\hline
\end{tabular}

Solo resulta obligatoria y suficiente la presencia de tres unidades: el núcleo propiamente dicho, la partícula como y el término. Mientras el núcleo excluye forzosamente la actualización con valor identificador, en el término impera esta 
actualización, ya sea explícita o implícitamente. En estrecha relación con esto, se observó en la muestra una prevalencia casi absoluta del sustantivo común como constituyente nuclear de la secuencia y del sustantivo propio como término de ella.

Se comprobaron dos restricciones morfosintácticas fundamentales para las unidades que integran las secuencias: la actualización -léxica, implícita o meramente morfemática- no identificadora del núcleo y la concordancia -sobre todo numéricaentre el núcleo y el término.

Las secuencias de como encontradas en la muestra que se ajustan de manera más incontrovertible a las ponderadoras de identificación son las que tienen por núcleo un sustantivo común en singular, actualizado por el indefinido un/una, aquellas en las cuales el indefinido algo desempeña función nuclear y las de núcleo compuesto por un sustantivo en plural, actualizado por el numeral cardinal dos.

Será necesario continuar profundizando en el tema, especialmente en la descripción de las diferencias morfosintácticas, semánticas y de orden pragmático entre las secuencias ponderadoras de identificación y aquellas otras que pudieran confundirse con ellas, en tanto poseen carácter ponderador y sus constituyentes se atienen a la misma estructura invariable y observan iguales restricciones morfosintácticas, pero resulta engorroso dirimir el alcance de la referencia del enunciado donde se insertan, sobre todo en dos casos fundamentales:

1. Cuando el núcleo aparece en plural y se halla inactualizado léxicamente o lo está por el indefinido de la serie otro/a y, a su vez, el término está constituido por una enumeración en que se incluye el nexo copulativo y como elemento de cierre y se produce cierta ambigüedad, similar a la de otros contextos de la lengua donde la conjunción $y$ es intercambiable por la conjunción $o$ (y viceversa) en enumeraciones abiertas;

2. Cuando el sintagma encabezado por como presenta un solo sustantivo (o unidad equivalente), en singular o plural, y no hay una voluntad marcada del emisor por distinguir si la predicación afecta específica y exclusivamente a la unidad introducida por como o si la afecta de manera prototípica.

Este deslinde, sustentado teóricamente a partir de realizaciones concretas, permitirá situar las secuencias ponderadoras de identificación en su relación sistémica con usos de como en construcciones adscriptivas, ejemplificadoras y propiamente comparativas.

Al mismo tiempo, sería conveniente ampliar la muestra de modo que se incluyeran otras variedades nacionales de la lengua española para comprobar la productividad de esta construcción en nuestro idioma y arribar a conclusiones teóricas de fiabilidad y alcance mayores.

NORMAS. REVISTA DE ESTUDIOS LINGÜÍSTICOS HISPÁNICOS, NÚMERO 3 (AÑO 2013):

http://www.uv.es/normas

(ISSN 2174-7245) 


\section{REFERENCIAS BIBLIOGRÁFICAS}

ACÍN ViLlA, Esperanza (1987): «Construcciones con como en español moderno», RILCE, III, 1, 25-57.

AlarCOS LlORACH, Emilio (1984): Estudios de gramática funcional del español, (3. ${ }^{a}$ edición), Madrid, Editorial Gredos (1. a edición, 1970).

Alarcos Llorach, Emilio (1994): Gramática de la lengua española, Madrid, Espasa Calpe, S.A.

AlciNA, Juan y J. Manuel BlecuA (1994): Gramática española (1056-1081), Barcelona, Ariel (1. a edición, 1975).

Bello, Andrés (1983): Gramática de la lengua castellana (con notas de R. J. Cuervo), La Habana, Editorial Pueblo y Educación.

CANO AguiLAR, Rafael (1995): Sintaxis histórica de la comparación en español, La historia de cómo, Sevilla, Secretariado de Publicaciones de la Universidad de Sevilla.

Cifuentes HonRubia, José Luis (1989): Lengua y espacio. Introducción al problema de la deíxis en lengua española, Alicante, Secretariado de Publicaciones de la Universidad de Alicante línea]: <http://publicaciones.ua.es/filespubli/pdf/LD84868096811847057.pdf> (también en <www.ua.es/personal/cifu/publicaciones/lenguayespacio.pdf>).

Cifuentes HonRubia, José Luis (2002): «Sobre sintagmas sustractivos, preposiciones, focalizadores y marcadores discursivos», Estudios de Lingüística, 16, 559 [en línea]: <www.ua.es/personal/cifu/publicaciones/Ssustrac.pdf>.

Cifuentes HonrubiA, José Luis (2004): «Sintagmas nominales encabezados por como, preposiciones y locuciones prepositivas», en Almela, Ramón et al., coords., Homenaje al profesor Estanislao Ramón Trives, Murcia, Universidad de Murcia, t. I, 175-186 (consultado en una versión digital facilitada por el autor).

Cuervo, Rufino J. (1998): Diccionario de construcción y régimen de la lengua castellana, Instituto Caro y Cuervo, Bogotá, Colombia, Instituto Caro y Cuervo, t. II, 222-236 (edición príncipe, 1954):

ESCANDELL, M. Victoria y Manuel LEONETTI (2000): «Categorías funcionales y semántica procedimental», en Martínez, M. et al., eds., Cien años de investigación semántica, de Michel Bréal a la actualidad, Madrid, Ed. Clásicas, vol. 1, 363-378 [en línea]: <www.uned.es/dpto-leng-esp-y-ling.../escandell/.../FuncyProc.PDF>.

EsCANDELL, M. Victoria y Manuel LEONETTI (2004): «Semántica conceptual/semántica procedimental», Actas del V Congreso de Lingüística General. Madrid, Arco [en línea]: <www.uned.es/dpto-leng-esp-y.../conceptualprocedimental.PDF>. 
GILI Y GAYA, Samuel (1975): Curso superior de sintaxis española. (4. ${ }^{a}$ edición), La Habana, Editorial Pueblo y Educación (1. a edición, 1943).

GUTIÉRREZ ORDÓÑEZ, Salvador (2000): «¿Qué hace un chicarrón como tú en un sitio como éste?», en Carbonero, Pedro et al., coords., Lengua y discurso. Estudios dedicados al Profesor Vidal Lamíquiz, Madrid, Arco Libros, 467-481 (facilitado digitalmente por el autor. Una versión de este artículo aparece incluida en el libro Forma y sentido en sintaxis, publicado en el 2002 por la propia editorial). Gredos.

HJElsmlev, Louis (1971): Prolegómenos a una teoría del lenguaje, Madrid,

IGLESIAS BANGO, Manuel (2006): «Categoría y transposición en el relativo como», en Villayandre, Milka, ed., Actas del XXXV Simposio Internacional de la Sociedad Española de Lingüística, Universidad de León [en línea]: $<$ http,//www3.unileon.es/dp/dfh/SEL/actas.htm>.

JiMÉNEZ JULIÁ, Tomás (1991): «Elipsis nominal y no realización en español», en Brea, Mercedes y Francisco Fernández Rei, coords., Homenaxe ó profesor Constantino García, Santiago de Compostela, Universidade de Santiago de Compostela, t. I, 223241 (versión digital facilitada por el autor).

JiMÉNEZ JULIÁ, Tomás (2003): «Como en español actual», Verba, 30, 117-161 (versión digital facilitada por el autor).

LYONS, John (1973): Introducción en la lingüística teórica, Barcelona, Editorial Teide (2. ${ }^{a}$ edición).

MARTí SÁNCHEZ, Manuel (1996): «Homogeneidad y heterogeneidad del sentido, el caso de cómo», Epos, XII, 131-157. Una versión de este artículo aparece incluida en Martí, Manuel y J. Miguel Hernández (1999), Indagaciones sobre conectores (capítulo 4), Córdoba, Ediciones Duque.

MARTíneZ, J. Antonio (1994a): Funciones, categorías y transposición, Madrid, Istmo.

MARTíNEZ, J. Antonio (1994b): Cuestiones marginadas de gramática española, Madrid, Istmo.

MONTORO DEL ARCO, Esteban T. (2005): Aproximación a la historia del pensamiento fraseológico español, las locuciones con valor gramatical en la norma culta (tesis doctoral de la Universidad de Granada) [en línea]: <http://digibug.ugr.es/bitstream/10481/677/1/15476893.pdf>.

MOYa CORRAL, J. Antonio (1999): «La oración compleja, los inclusores», Actas de la II Conferencia de Hispanistas de Rusia, Madrid, Embajada de España en Moscú, Ministerio de Asuntos Exteriores [en línea]: $<$ http://hispanismo.cervantes.es/documentos/moyacorral.pdf $>$. 
PAVÓN LUCERO, M. Victoria (1999): «Clases de partículas, preposición, conjunción y adverbio», en Bosque, Ignacio y Violeta Demonte, coords., Gramática descriptiva de la Lengua Española, Madrid, Espasa Calpe, 565-655.

POTTIER, Bernard (1977): Introducción al estudio de la morfosintaxis española, La Habana, Edición Revolucionaria. [Esta edición es tomada de la original, publicada en 1959. No se consigna la casa editora]

REAL ACADEMIA ESPAÑOLA Y ASOCIACIÓN DE ACADEMIAS DE LA LENGUA ESPAÑOLA (2010): Nueva gramática de la lengua española, Madrid, Espasa Libros.

Textos literarios ${ }^{13}$

Alguien

Buenos

Cándido

Bobes, Marilín (2001): Alguien tiene que llorar, La Habana, Ediciones Unión. Col. de cuentos, 105 pp.

Riverón, Rogelio (2000): Buenos días, Zenón, La Habana, Ediciones Unión. Col. de cuentos, 113 pp.

Diálogos

PÉreZ, Jorge Ángel (2001): Cándido habanero, D.F., México, Editorial Colibrí. Novela (Publicada en Cuba el propio año 2001 por la editorial Unión, bajo el título El paseante Cándido), 199 pp.

Fumando

Araúso, Nara. (2003): Diálogos en el umbral, Santiago de Cuba, Editorial Oriente. Col. de ensayos, 156 pp.

Hombre
Minuciosas

PÉREZ, Jorge Á. (2003): Fumando espero, La Habana, Editorial Letras Cubanas. Novela, 235 pp.

CABAllero, Rufo. (2005): Un hombre solo y una calle oscura, La Habana, Ediciones Unión. Ensayo, 160 pp.

Noche ARRUFAT, Antón. (2002), La noche del aguafiestas, La Habana, Editorial Letras Cubanas (1. ${ }^{a}$ edición, 2000, Editorial Letras Cubanas), Novela, $237 \mathrm{pp}$.

\footnotetext{
${ }^{13}$ Las citas de textos literarios que forman parte de la muestra se refieren en el cuerpo del trabajo escribiendo entre paréntesis la palabra que identifica la obra y luego la página del texto donde aparece. Ejemplo: Saturno 45, indica que la cita es tomada del libro Saturno en el espejo, de Luis Álvarez, página 45 .
} 
Presunciones

Saturno
GARRAndÉs, Alberto. (2005): Presunciones, La Habana, Editorial Letras Cubanas. Col. de ensayos, 410 pp.

Álvarez, Luis. (2004): Saturno en el espejo, La Habana, Ediciones Unión. Ensayo, 215 pp.
Textos periodísticos ${ }^{14}$

Escambray, abril

Escambray, mayo

Escambray, junio

Granma, abril

Granma, mayo

Granma, junio

Juventud
Escambray, abril 2008 (días 5, 12 y 19), 24 pp.

Escambray, mayo 2008 (día 24), 8 pp.

Escambray, junio 2008 (día 28), 8 pp.

Granma, abril 2008 (días 7, 8, 9, 11, 12, 14 y 26), 64 pp.

Granma, mayo 2008 (días 6,9, 14, 16,20, 22,23, 26,27,31), 104 pp.

Granma, junio 2008 (días 5, 6, 10, 12, 13, 16), 64 pp.

Juventud Rebelde, 18 de marzo de 2008, 8 pp

\footnotetext{
${ }^{14}$ Las citas de textos periodísticos que forman parte de la muestra se refieren en el cuerpo del trabajo escribiendo entre paréntesis la palabra que identifica a la publicación, luego el mes con indicación del día y por último la página donde aparece. Ejemplo, Escambray, abril 8: 5, indica que la cita es tomada del periódico Escambray, de abril de 2008, día 8, página 5. En el caso de los decursos extraídos de Juventud Rebelde, las citas se harán siguiendo el procedimiento para los textos literarios, solo se consigna la palabra y la página donde aparece.
}

NORMAS. REVISTA DE ESTUDIOS LINGÜÍSTICOS HISPÁNICOS, NÚMERO 3 (AÑO 2013):

http://www.uv.es/normas

(ISSN 2174-7245) 
